\title{
Impact of tacrolimus trough level at discharge on acute rejection rate in sensitized renal transplant recipients: a national cohort study
}

\author{
Changsin Lee ${ }^{1}$, Jun Gyo Gwon ${ }^{1}$, Myung Gyu Kim² ${ }^{2}$ Cheol Woong Jung ${ }^{1}$
}

${ }^{1}$ Department of Surgery, Korea University Anam Hospital, Seoul, Korea
${ }^{2}$ Department of Internal Medicine, Korea University Anam Hospital, Seoul, Korea

Background: Tacrolimus (TAC) is one of the most important maintenance immunosuppressants in kidney transplantation (KT) and maintaining the proper blood concentration is essential to prevent acute rejection. Several studies have been published on appropriate TAC blood concentrations, but the timing of measurement and the proposed blood concentrations vary from study to study. Moreover, an optimal TAC trough level is rarely presented in sensitized patients who are prone to rejection. This study was to analyze the association between TAC trough level at discharge and subsequent acute rejection rates.

Methods: We analyzed a total of 4,001 KTs collected in Korea Organ Transplantation Registry between April 2014 and December 2019. A total of 1,504 sensitized recipients (defined as crossmatch positive, panel reactive antibody $\geq 10 \%$, or donor-specific human leukocyte antigen antibodies positive) were enrolled, and they were divided into low-level ( $\leq 5.9 \mathrm{ng} / \mathrm{mL}$; less than 25 th percentile), mid-level ( $>5.9 \& \leq 9.5 \mathrm{ng} / \mathrm{mL}$ ) and high-level ( $>9.5 \mathrm{ng} / \mathrm{mL}$; over 75 th percentile) groups according to TAC trough level at discharge. Acute rejection rate within 1 year after KTs and graft function were evaluated.

Results: There was no statistical difference in baseline characteristics of the three groups. The incidence of biopsy-proven acute rejection (BPAR) was not significantly different between the three groups, although there was a tendency of increasing the rejection rates in lower TAC trough level groups compared with high-level group ( $9.1 \%$ vs. $7.3 \%$ vs. $6.3 \%$; $P=0.316)$. Estimated glomerular filtration rate at 1 year after KTs also was not significantly different between the groups.

Conclusions: This study suggests that maintaining a higher TAC trough level at the time of discharge in sensitized KT recipients might reduce the incidence of BPAR within 1 year after KTs.

Corresponding author: Changsin Lee

E-mail:viano0420@naver.com

(c) The Korean Society for Transplantation

This is an Open Access article distributed under the terms of the Creative Commons Attribution Non-Commercial License (http://creativecommons.org/licenses/by-nc/4.0/) which permits unrestricted non-commercial use, distribution, and reproduction in any medium, provided the original work is properly cited. 